\title{
El papel de la ética en la enseñanza de lenguas ${ }^{1}$
}

\section{(The Role of Ethics in Language Teaching)}

\section{Luis Guillermo Barrantes Montero 2}

\author{
Universidad Nacional, Costa Rica
}

\begin{abstract}
resumen
Se propone el fundamento de la dimensión ética en la enseñanza de lenguas. Se señalan las divergencias en el ethos de las culturas moderna y postmoderna para contextualizar el tema. A partir de ello se resalta la condición de persona como valor máximo a cuya promoción integral han de orientarse las diversas acciones humanas. Dado que la enseñanza de lenguas implica una confrontación entre la mismidad y la otredad, resulta imperioso recuperar su identidad como disciplina humanística -más allá del instrumentalismo positivista propio de la expansión del capitalismo económico financiero- con el fin de cimentar la valorabilidad ética de las acciones que se toman en ella.
\end{abstract}

\section{abstract}

An attempt is made to provide a basis for the ethical dimension of language teaching. Discrepancies about ethos in modern and post-modern cultures are discussed to contextualize the topic. That will be the starting point to give the person as such the highest value, toward which all integral development of human actions must be oriented. Since language teaching

1 Elaborado con base en la ponencia presentada por el autor en el IV Congreso Internacional de Lingüística Aplicada, llevado a cabo en mayo de 2013, en el Campus Omar Dengo, de la Universidad Nacional de Costa Rica. Recibido: 29 de abril de 2012; aceptado: 3 de agosto de 2012.

2 Escuela de Literatura y Ciencias del Lenguaje. Correo electrónico: luisba66@yahoo.es

$$
L_{\text {etras }} 52 \text { (2012), ISSN 1409-424X }
$$


implies a confrontation between sameness and otherness, it is crucial to recover its identity as a humanistic discipline-beyond the positivistic instrumentalism characteristic of current financial economic capitalism-with the aim of establishing the ethical value of any actions taken within its scope.

Palabras clave: ética, educación, enseñanza de idiomas, deontología Keywords: ethics, education, language teaching, deontology

\section{Introducción}

Es interesante constatar que, si se le pregunta a un docente de lenguas sobre qué tiene que ver la ética con esa rama de la lingüística aplicada, probablemente responderá que «mucho». Sin embargo, si el escrutinio va un poco más allá y se le interroga por su referente ético o por la razón de ser de las normas que conoce, quizá se mostrará un tanto vacilante. Si bien el tema ético concierne, de un modo u otro, a cualquier campo del saber (y del actuar), este tema no parece ser prioritario en el pensum de estudio de las carreras universitarias $y$, por ende, los profesionales en ejercicio adolecen de serias debilidades en cuanto a su formación deontológica.

El presente estudio aborda el tema de la ética de los profesionales en la enseñanza de lenguas, pero no desde un ángulo tradicional y ampliamente conocido: el de analizar la medida en que determinadas acciones se ajustan a determinadas normas; ni siquiera se pretende enfatizar la reflexión de por qué una norma está justificada o no. Se busca, más bien, en un primer momento, poner de manifiesto el tipo de sociedad en la que se mueven los docentes, en tanto sujetos responsables de su actuar. Por ello se plantea el problema del cambio de paradigmas al que asiste tal sociedad. En un segundo momento, se propone la persona como referente ético en diálogo con las diversas cosmovisiones que conviven hoy en un mundo cada vez más interconectado gracias a las tecnologías de la información. En tercer lugar, se hace hincapié en la formación de la consciencia ética como condición para que las decisiones de las personas estén encaminadas 
a objetivos de mejores modos de convivencia entre la gente y de armonía de esta con su entorno. Un cuarto aspecto es la enseñanza de lenguas en cuanto disciplina como objeto de reflexión epistemológica, a fin de recalcar que el cometido último de ese quehacer trasciende la simple práctica docente y busca un ejercicio profesional con criticidad. Finalmente, se recapitula mediante el señalamiento de lo que debería subyacer en la cosmovisión de los profesionales en la enseñanza de lenguas, de acuerdo con lo que reclama en la actualidad el Zeitgeist o espíritu de los tiempos.

\section{Aclaración de conceptos}

En vista de que los destinatarios de esta disertación son profesionales académicos con especialidad en lingüística aplicada y otras disciplinas afines, conviene aclarar cierta terminología que, si bien suele ser comúnmente empleada, no pertenece a los ejes centrales de esas materias y, por tanto, son susceptibles a sesgos e imprecisiones.

En primer lugar, no sorprende que haya confusión en cuanto a los conceptos de moral y ética. El primero de ellos, según la tradición filosófica occidental, se refiere al conjunto de normas, costumbres y pautas de comportamiento que una sociedad acepta y por las cuales se rige; mientas que el segundo es un proceso que implica mayor conciencia y racionalidad: se trata de una reflexión acerca de esas normas. En palabras de Pérez y Velasco33; «la moral pregunta qué debo hacer (hábitos, costumbres). La ética pregunta por qué debo hacerlo; se sitúa, así, en un nivel superior de profundidad. El por qué es una pregunta por la fundamentación de las morales, es una pregunta por su razón de ser».

$\mathrm{Al}$ afirmar que la ética requiere de más racionalidad, se entiende que cierta costumbre o vivencia cotidiana (objeto de la moral) puede ser adoptada e, incluso, convertida en hábito sin que, de modo

3 Elizabeth Pérez y José A. Velasco, «Variaciones sobre el concepto de ética», Crea Ciencia IV, 7 (2007): 28-33. 
necesario, haya mediado una valoración consciente, o lo que se conoce como pre-juicio. Por eso se dice que una persona es (o no es) ética, mientras que un acto es (o no es) moral. En otras palabras, la moralidad es la medida en que las acciones humanas se ajustan a las normas establecidas por la sociedad, estén o no consideradas dentro de la legislación o normativa de determinada sociedad. Por su parte, la ética tiene que ver con la calidad humana de la gente.

En segundo lugar, la deontología es la disciplina que trata de los deberes de los profesionales ${ }^{4}$. En el sexto apartado de esta ponencia se desarrolla el tema de forma más prolija. Baste por ahora tenerlo presente en cuanto valoración ética de las acciones de quienes ejercen determinada disciplina.

En tercer y último lugar, valga la distinción entre persona e individuo, dentro del pensamiento occidental tradicional. Puesto que ambos términos a menudo se usan como sinónimos, resulta útil precisar que persona designa al individuo en cuanto ser dotado de espiritualidad y consciente de su ser-en-relación. En ello estriba el fundamento de la dignidad humana. Como lo apunta Emery, citando al Aquinate: «Debido a que subsistir en una naturaleza racional es una gran dignidad, a cada individuo de naturaleza racional se le llama persona ${ }^{5}$.

Aclarada la terminología, podríamos abordar, sin más, el tema que nos ocupa: la ética en la enseñanza de lenguas. No obstante, eso no parece ser tan sencillo: ya entrado el segundo decenio del siglo xxi no es posible tratar casi ningún tema, de manera plena y exhaustiva, si se deja de lado el fenómeno de la postmodernidad. En el caso de la ética, no se da una excepción.

4 Hugo Álvarez Martínez, «La intimidad asediada: psicoanálisis, deontología y cultura», Revista de Psicología y Ciencias afines, VII, 11 (2010): 58-65.

5 Gilles Emery, The Dignity of Being a Substance: Person, Subsistence and Nature [La dignidad de ser sustancia: persona, subsistencia y naturaleza], Nova et Vetera (English Edition) IX, 4 (2011): 991-1001 (1001). Traducido por el autor a partir de la fuente en inglés. La cita original de Santo Tomás es «Et quia magnae dignitatis est in rationali natura subsistire, ideo omne individuum rationalis naturae dicitur persona.» (ST, q. 29.a. 3,ad 2). 


\section{El ethos en la era postmoderna y transmoderna}

La crisis del modernismo positivista y su seguridad en las posibilidades de la ciencia, la técnica, el derecho y la política trajo como resultado, a finales del siglo xx, una sensibilidad (y un tipo de sociedad), caracterizada por la renuncia a todo dogmatismo y a las grandes metanarrativas. Estas limitaban la capacidad de discrepar y de cuestionar, todo mediante la opresión cultural y el miedo a pensar. La irrupción de lo postmoderno minó muchas seguridades: en vez de mantener la confianza en el conocimiento y en los sistemas ya construidos surge un interés por la de-construcción, por el cuestionamiento y por la puesta en evidencia de lo que antes se mantenía oculto. Cobra relevancia el contexto, la localidad, lo coyuntural y la perspectiva ecológica.

El tema ético cobra interés en el ambiente postmoderno. Dos destacados pensadores, Jürgen Habermas y Jean-François Lyotard, coinciden en que esta sería época de emancipación del ser humano, de la sociedad y de la naturaleza misma. Sin embargo, el primero abogaba por el consenso para «ordenar racionalmente el mundo, en libertad $»^{6}$; Lyotard, al principio se inclina por el disenso y la relativización de toda propuesta como camino a la emancipación; en un segundo momento, considera que la manera de resistir a la o presión intelectual y cultural es la reescritura de «todo aquello que pueda cambiarse para transformar la opinión general» ${ }^{7}$. En este contexto, Guerrero realiza un aporte a la pedagogía que considera emancipatoria y puede iluminar el ethos de la enseñanza de lenguas en nuestro tiempo ${ }^{8}$ : «La nueva pedagogía emancipatoria reconocerá que la última palabra ha de ser que cada persona tenga derecho a ser un sujeto que aspire a ser libre en todos los ámbitos de su vida personal y colectiva».

\footnotetext{
6 Antonio Guerrero, «Condición postmoderna y esbozo de una nueva pedagogía emancipatoria. Un pensamiento diferente para el siglo xxi », Revista de Estudios Sociales XLII, 42 (2012): 27-39.(30).

7 Guerrero, 31.

8 Guerrero, 37.
} 
En la misma línea, Boaventura de Sousa Santos, en su Crítica de la razón indolente ${ }^{9}$, plantea la cuestión modernidad/postmodernidad como confrontación entre el paradigma dominante (proveniente de la mentalidad modernista-positivista y que ha sido el caballo de batalla de la globalización) y el emergente (la nueva sensibilidad que aspira a la emancipación). Según el paradigma dominante, el conocimiento «objetivo», el de la ciencia, desdeña y subestima el conocimiento que surge del sentido común. Se debe rescatar, más bien, el conocimiento que, gracias a un nuevo sentido común emancipador, resulte obvio y útil: «El conocimiento-emancipación tiene que convertirse en un sentido común emancipador: imponiéndose al prejuicio conservador y al conocimiento prodigioso e impenetrable, tiene que ser un conocimiento prudente para una vida decente».

Así, también se impone un nuevo sentido común ético. El paradigma dominante opera mediante una ética antropocéntrica e individualista, que dicta normas morales prácticas, según el criterio de la ciencia y la tecnología (y de acuerdo con la fórmula sujeto-acciónconsecuencia) pero niega a la gente común la posibilidad de asumir el principio de la responsabilidad. En la época en que vivimos, sin embargo, no es tan sencillo determinar quiénes son los agentes, a qué obedecen sus acciones ni cuáles son las consecuencias. Por eso la ética del nuevo sentido común está inspirada en la solidaridad. Al contrario del antropocentrismo, individualismo e inmediatez de la ética liberal, esta nueva ética consiste en una preocupación por el individuo, la comunidad y el entorno, cuya existencia debe estar garantizada desde hoy. En eso consiste el principio de la responsabilidad.

Un aspecto que aborda Santos, de relevancia en nuestra disertación por estar relacionado con la deontología en la enseñanza de lenguas, tiene que ver con la actitud en favor de la justicia cultural. Ante el reconocimiento de que todas las culturas son relativas e incompletas y de que existe gran diversidad de las mismas, urge hacer relecturas

9 Boaventura de Sousa Santos, Crítica de la razón indolente (Bilbao: Desclée de Brower, 2003) 121. 
recíprocas de aquello que las fundamenta para alcanzar un enriquecimiento también recíproco. Santos le llama a ello la hermenéutica diatópica, según Santos, citado por Vergalito ${ }^{10}$ : «El objetivo de la hermenéutica diatópica no es (...) lograr la completud -lo que es considerado como un fin inalcanzable- sino, por el contrario, suscitar la conciencia de la incompletud recíproca tanto como sea posible, mediante la participación en el diálogo de la manera que se haría si se tuviera un pie en una cultura y otro en otra. De ahí su carácter diatópico». Lo propuesto por Santos no escapa de críticas en cuanto a su viabilidad. Aun así, se ha citado aquí para ilustrar el tema que se discute.

Puede que los jóvenes que asisten a las universidades e incluso los mismos docentes no estén familiarizados con este tipo de debates. Es un hecho que nadie puede abstraerse de la influencia de la sociedad y de la coyuntura histórica en que se vive. La sensibilidad postmoderna está presente en las inquietudes y los reclamos de la juventud. Achacar el problema de la escasa comunicación y la brecha generacional a una supuesta «pérdida de valores», como frecuentemente se oye decir, no es más que una manera simplista de entender lo que ocurre. El objetivo de plantear la cuestión ética tanto desde el ángulo de la tradición occidental como desde el postmoderno consiste, precisamente, en suscitar el interés de los académicos en el asunto y de que, de algún modo, se involucren en esta transición paradigmática.

Por su parte, Dusse $1^{11}$ denomina «transmodernidad» a «todos los aspectos que se sitúan "más-allá" (y también "anterior") de las estructuras valoradas por la cultura moderna europeo-norteamericana, y que están vigentes en el presente en las grandes culturas universales no-europeas y que se han puesto en movimiento hacia una utopía pluriversa». Una ética desde la transmodernidad, es una ética en diálogo intercultural. Toma en cuenta las visiones de las culturas

10 Esteban Vergalito, «Acotaciones filosóficas a la "Herméutica Diatópica" de Boaventura de Sousa Santos», Impulso XIX, 48 (2009) 19-29 (21).

11 Enrique Dussel, Transmodernidad e interculturalidad. Interpretación desde la Filosofía de la Libración (2005), 18; <http://www.olimon.org/uan/transmodernidad.pdf>, 31 de marzo de 2013. 
que por siglos han permanecido eclipsadas por la visión de la modernidad dominante y las sociedades hegemónicas que la han empleado como su caballo de batalla.

En el campo de la enseñanza de lenguas, la transmodernidad supone un particular interés ya que obliga a los involucrados en el proceso educativo a tener en cuenta los paradigmas locales y la visión del mundo de cada lugar donde se enseña y aprende una lengua extranjera para que se realice, de manera continua, una autocrítica y una revisión de valores y antivalores tanto de la propia sociedad como de aquella que se está conociendo mediante el estudio de su lengua. Esto enriquece la experiencia ética y cultural de las sociedades y puede llevar a un mundo más tolerante, inclusivo y solidario.

\section{La persona como referente ético}

Proponer a la persona como referente ético requiere de precaución. Dado que el concepto de persona ha sido empleado y manipulado a lo largo de la historia por los poderes hegemónicos, a fin de preconizar determinadas ideas que defienden intereses antropocéntricos, religiosos, occidentalistas y de otros sesgos muy variados y, que a su vez, marginan otras realidades y modos de ser alternativos a los que acepta el paradigma dominante, hay que desarraigar ese concepto de los aditamentos semánticos que se le han ido adhiriendo.

En el seno mismo del cristianismo, en que la doctrina personalista ha sido clave, hay tendencias que concuerdan con la cosmovisión postmoderna. Para quienes así piensan, ya es insostenible la fe basada en la idea de que el mundo que conocemos depende de otro mundo que existe en un nivel superior, es decir, una fe heterónoma. Al contrario, consideran que Dios es el núcleo creador del proceso cósmico. Esta cosmovisión autónoma la entienden, más bien, como 
teónoma, pues reconcilia la autonomía humana con la fe en Dios. Lenares $(2008: 26)^{12}$ lo expresa como sigue:

Desde el momento en que no exista una instancia exterior y superior al cosmos, que todo lo sabe y conoce y que se digna comunicar algo de esto a un determinado número de elegidos, entonces todo lo que pensamos sobre «Dios» o lo que se refiere a Él, proviene de nuestro pensamiento, intuición y búsqueda, y se halla en una evolución constante. Entonces, cada expresión es hija de su tiempo y exhibe rasgos de este padre inquieto y contradictorio. En otras palabras, las formulaciones son verdaderas y buenas sólo hasta un cierto grado, en la medida en que derivan del punto de partida escogido y son consecuentes con él. No pueden trasmitir una experiencia de la realidad, sino hasta donde ella ha sido recogida por seres humanos -esto es lo que significa la verdad. Por eso, cualquier expresión puede ser revisada y mejorada a lo largo del tiempo.

Volviendo a la transición paradigmática, es posible considerar al ser humano, a la persona, como referente ético, desnudado y rescatado de todas las connotaciones alienadoras que ha tenido y se abre un espacio al diálogo intersubjetivo, en el que pueden hacer sus aportes todos aquellos que comparten el anhelo de libertad, de placer $^{13}$, de solidaridad y de compromiso con el futuro.

\section{De la moral heterónoma a una moral autónoma mediante la for- mación de estados de consciencia}

A la luz del cambio de paradigmas presentados en este trabajo, se comprende que el ser humano de hoy ya no está conforme con una moralidad consistente en el simple ajuste de sus actos con las normas dadas por algún referente heterónomo. El tema de la moral podría ser

12 Roger Lenares, s.j. Otro cristianismo es posible. Fe en lenguaje de modernidad (Quito: Abya Yala, 2008).

13 Edilene Queiroz, «El dolor y el gozo: de Freud a Lacan», Revista latinoamericana de Psicopatología fundamental XV, 4 (2012): 851-867, 858. 
indiferente en muchas personas; sin embargo, en razón de encontrarnos involucrados en la transición paradigmática, una cierta actitud escéptica, al menos temporalmente, puede ser comprensible. Por ello la pedagogía se ubica en una posición estratégica como medio para el alcance de estados de consciencia más elevados por parte de las nuevas generaciones, a fin de que abracen su responsabilidad con la construcción de una vida decente ${ }^{14} y$, si se quiere, con unateonomía ${ }^{15}$.

Desde la perspectiva psicoanalítica, la ética pedagógica consiste en permanecer abiertos a lo Real ${ }^{16}$, es decir, a los eventos de la cotidianidad para los que no estamos preparados, y afrontarlos con conciencia de lo que invertimos en ello, sea a nivel psíquico o a nivel de nuestros gustos y preferencias. Es, en última instancia, actuar en consecuencia con los dictámenes de esa consciencia. Taubman ${ }^{17}$ lo propone así: «La ética pedagógica que propongo aquí nos pide afrontar nuestros propios deseos, arriesgar nuestra seguridad y las nociones de lo que somos, explorar nuestra propia complicidad en lo que nos acontece y mirar con atención nuestras historias, individuales y colectivas».

Si hablamos de la deontología en la enseñanza de lenguas, el punto de partida debe ser el reconocimiento de que cuanto mayor sea el estado de consciencia del ser humano, con mayor plenitud desarrollará su vocación de ser persona, en el sentido antes expuesto y, por ende, también crecerá su potencial de contribuir, solidariamente, con la exaltación del valor de los demás seres humanos y del entorno inmediato y global.

\section{La enseñanza de lenguas y su marco epistemológico}

La epistemología es la vertiente filosófica que se ocupa de la estructura, el fundamento y los métodos desarrollados por los sistemas

\footnotetext{
14 Santos (2003) 116.

15 Lenares, 26.

16 Peter Taubman, «Alain Badiou, Jacques Lacan and the Ethics of Teaching» [Alain Badiou, Jacques Lacan y la ética de la enseñanza], Educational Philosophy and Theory XLII, 2 (2010): 196-212 (196).

17 Traducido por el autor; Taubaum, 209.
} 
científicos ${ }^{18}$. La enseñanza de idiomas, como parte de la lingüística aplicada, también es objeto de análisis epistemológico. Sin embargo, este trabajo mantiene la perspectiva del cambio de paradigmas al que asistimos en la actualidad. Por ello, no basta con hablar de epistemología sino de epistemologías. En este sentido se presenta la epistemología de la educación, tanto desde el ángulo moderno como desde el postmoderno. Con respecto al primero, De Becerra ${ }^{19}$ nos dice lo siguiente:

La enseñanza desde la postura moderna o tradicional, sustentada en el positivismo, se caracteriza, entre otros, por los siguientes aspectos: a) adopta como válido para todas las ciencias solo el método científico; b) el conocimiento es un reflejo, en el sujeto, de algo que está fuera (el objeto), el investigador no interfiere con su objeto de estudio; c) la ciencia avanza inductivamente por acumulación de verdades objetivas, de lo observable, de lo inmediato, de los hechos a la teoría; d) el progreso de la ciencia es continuo, se basa en verdades verificadas y ciertas sobre los hechos naturales y sociales.

El aspecto más inquietante, para la enseñanza de lenguas, se refiere a la no interferencia del investigador con su objeto de estudio. En ese caso podrá calcularse cuánto más distante estará el docente de lenguas de esa interferencia. Si su pedagogía se basa en el estricto seguimiento de determinado libro de texto, la visión del mundo y de la cultura extranjera que adquieren sus estudiantes tendrá sesgos importantes.

Desde lo postmoderno, la epistemología pedagógica reviste otra identidad más a tono, si se quiere, con las perspectivas emancipadoras que se han estado planteando. Al respecto, De Becerra ${ }^{20}$ afirma:

En la postmodernidad, la ciencia no es un proceso acumulativo y lineal. Presenta rupturas y discontinuidades, por la aparición de

18 Pérez y Velasco, 28.

19 Gloria De Becerra, «Enseñanza de la investigación y epistemología de los docentes», Educación y educadores XI, 2 (2008): 61-68 (63).

20 De Becerra, 63. 
nuevos marcos conceptuales y por efecto de nuevas estrategias para conocer (Khun, 1992). La visión postmoderna valida las subjetividades, las emociones, las necesidades especiales, las ideas previas, el nivel conceptual de quienes aprenden. El proceso enseñanzaaprendizaje es dialéctico, tanto el docente como el estudiante, como unidad dialéctica, tienen la posibilidad de aprender mutuamente.

La importancia de tener presente el marco epistemológico de la enseñanza de lenguas en la búsqueda de un principio y fundamento deontológico para sus profesionales estriba, pues, en que no basta que estos conozcan el objeto material de esta rama de la lingüística aplicada, sino que, al tener igualmente claro su objeto formal, es decir, la perspectiva desde la que se aborda, podrán distinguir mejor lo que es ético de lo que no lo es dentro de su disciplina.

\section{Deontología en la enseñanza de lenguas}

La deontología es el estudio de la exigencia ética de los profesionales. Expuestas las cuestiones referentes a la eticidad en la transición de paradigmas y el enfoque epistemológico de la enseñanza de lenguas, se puede apuntar, de forma más precisa a los deberes éticos del docente de lenguas y, extensivamente, del investigador. Las claves éticas del profesional en enseñanza de lenguas, en consonancia con lo que se ha planteado hasta ahora, van en la línea del reconocimiento intersubjetivo, la actitud solidaria y la preocupación por contextualizar las experiencias de aprendizaje.

En cuanto a lo primero, el profesional en enseñanza de lenguas desarrolla la sagacidad de interpretar las intencionalidades patentes y latentes en sí mismo, en sus alumnos, en el currículo, en los materiales de estudio y en las políticas institucionales ${ }^{21}$. Esta apertura interactiva a la intersubjetividad y a la interculturalidad le ayudará

21 Carmen Ortiz y otros, «Curriculum y docente: encuentro de significados», Revista cubana de educación médica superior XXII, 2 (2008): 1-6 (6). 
a tomar decisiones juiciosas en beneficio de su labor docente. Con respecto a lo segundo, la ética del docente lo sitúa como formador de opinión y de sentido de responsabilidad, por el cual las personas son interpeladas a tomar partido por sí mismas, por sus prójimos, por la naturaleza y por el futuro «cuya posibilidad de existencia debemos garantizar en el presente $»^{22}$. Y, en cuanto a lo tercero, se puede afirmar que una actitud ética en la enseñanza de lenguas puede hacer la diferencia entre perpetuar un transmisionismo que, en muchos casos, resulta alienante y una experiencia de enseñanza-aprendizaje que sea significativa y liberadora. Al respecto, Van Lier citado por Tudor ${ }^{23}$, afirma lo siguiente:

Una perspectiva ecológica de la enseñanza de lenguas ofrece un modo alternativo de ver los contextos en los que se ubican el uso y el aprendizaje de lenguas [...] Propone ser una alternativa radical al racionalismo cartesiano, al dualismo mente-cuerpo y al mundo antropocéntrico promovido durante siglos. Reemplaza esas ideas con una concepción del ambiente de aprendizaje como un complejo sistema de adaptación, de la mente como la totalidad de relaciones entre una persona que se desarrolla y el mundo circundante y del aprendizaje en cuanto actividad significativa en un ambiente accesible.

\section{El docente de lenguas y su Weltanschauung o visión del mundo}

La dimensión ética le exige al ser humano constantes tomas de decisión y definición de posturas definidas, tanto ante eventos cotidianos menores como ante los grandes dilemas de la vida. Es por ello que esta disertación ha puesto un cuidado especial en la presentación de diferentes ángulos de consideración para la toma de partido y también en la necesidad de desarrollar estados de consciencia que permitan decidir con propiedad.

22 Santos (2003) 126.

23 Ian Tudor, The Dynamics of the Language Classroom (Cambridge: Cambridge University Pres, 2001) 9. 
El docente de idiomas, en el siglo xxi, deberá ejercer su capacidad de toma de postura según la Weltanschauung o cosmovisión que haya desarrollado. Por una parte, la fuerte influencia que ejerce el capitalismo neoliberal, mediante las políticas de globalización, ha permeado los sistemas educativos, convirtiendo las universidades en centros de comercio de la educación como parte de los servicios que se pueden comercializar ${ }^{24}$. Muchos profesores convierten el afán globalizador en la brújula que marca el «norte» de su cosmovisión.

Por otra parte, quienes se conduzcan según los principios deontológicos anteriores, es decir, el reconocimiento intersubjetivo, la actitud solidaria y la preocupación por contextualizar las experiencias de aprendizaje, tendrán la posibilidad de contribuir con el nuevo sentido común para una vida decente ${ }^{25}$. Incluso, pueden utilizar los recursos tecnológicos y el potencial que ofrece la comunidad académica internacional para contribuir con una forma de globalización anti-hegemónica ${ }^{26}$. La Weltanschauung del docente de idiomas puede ser cultivada, pero incluso, el interesarse por ese cultivo también implica una opción.

\section{La ética y el zeitgeist o espíritu de los tiempos}

Se entiende por «Zeitgeist» o espíritu de los tiempos un conjunto de valores, ideas y suposiciones ${ }^{27}$ que una sociedad de determinada época llegar a asumir. Pero tal naturaleza, suele ser cambiante y, lo que se acepta sin dificultad durante un tiempo puede variar significativamente en otro. Así, la ética también debe estar atenta al

24 Roger Dale y Susan Robertson, «Interview with Boaventura de Sousa Santos» [Entrevista con Buenaventura De Sousa Santos], Globalization, Societies and Education II, 2 (2004): 148-160 (155). 25 Santos (2003).

26 Dale y Robertson, 160.

27 Karola Hahn, «The Changing Zeitgeist of German Higher education and the Role of GATS» [El cambiante Zeitgeist de la Educación Superior Alemana y el papel de los AGC (Acuerdos generales de Comercio y servicios), Higher Education in Europe XXVIII, 2 (2003): 199-219. 
espíritu de los tiempos para discernir los valores y antivalores de la sociedad y repensar sus criterios para tomar posición.

En la enseñanza de lenguas la fuerte influencia de la globalización puede hacer creer al académico que los planteamientos emanados desde las naciones hegemónicas mediante sus casas editoriales, congresos internacionales y «programas de cooperación» cultural y educativa pueden ser de una lógica innegable. No obstante, la realidad innegable de las fallidas promesas de bienestar para quienes aprenden una lengua extranjera, pueden suscitar nuevos hálitos de esperanza mediante una aproximación crítica a la tarea de enseñar y aprender un idioma.

\section{Conclusiones}

La ética en la enseñanza de lenguas en este momento de nuestro siglo no puede estar fundamentada en normas establecidas en condiciones coyunturales que no corresponden a las de hoy. La sociedad actual atraviesa un estado de transición paradigmática, debido a lo cual los referentes de todas las dimensiones de la vida, incluida la ética, se encuentran sometidos a escrutinio, a crisis y a relatividad.

La deontología en la enseñanza de lenguas debe fundarse en una apertura a la intersubjetividad y en una definición sólida de lo que implican la mismidad y la otredad cuando las diversas culturas se encuentran mediante el aprendizaje de sus idiomas. El docente de lenguas requiere una formación sólida en tres componentes clave, además del contenido propio de la disciplina, y son la base epistemológica de su campo de conocimiento, el pensamiento crítico y el forjamiento de un estado de consciencia lo suficientemente elevado como para permitirle tomar decisiones maduras y transmitir el mismo talante a sus educandos.

Queda pendiente, para ulteriores investigaciones, el estudio de casos concretos en que esta aproximación a la ética pedagógica puede implementarse en los planes de estudio y en la preparación de lecciones. 